\title{
Linear theory of microwave instability in electron storage rings
}

\author{
Yunhai Cai \\ SLAC National Accelerator Laboratory, Stanford University, Menlo Park, California 94025, USA
}

(Received 24 January 2011; published 14 June 2011)

\begin{abstract}
The well-known Haissinski distribution provides a stable equilibrium of longitudinal beam distribution in electron storage rings below a threshold current. Yet, how to accurately determine this threshold, above which the Haissinski distribution becomes unstable, is not firmly established in theory. In this paper, we will show how to apply the Laguerre polynomials in an analysis of this stability that are associated with the potential-well distortion. Our approach provides an alternative to the discretization method proposed by Oide and Yokoya. Moreover, it reestablishes an essential connection to the theory of mode coupling originated by Sacherer. Our new and self-consistent method is applied to study the microwave instability driven by commonly known impedances, including coherent synchrotron radiation in free space.
\end{abstract}

DOI: 10.1103/PhysRevSTAB.14.061002

PACS numbers: 29.27.Bd, 29.20.db

\section{INTRODUCTION}

The idea of using mode couplings to explain the instability of a bunch beam was first proposed and then studied over many years by Sacherer [1]. The theory essentially consists of an integral equation, known as the Sacherer integral equation, derived as a perturbed Vlasov equation, which is solved as an eigenvalue problem of a matrix. It was extended by Besnier [2] who introduced orthogonal polynomials to the theory. It was further developed by Zotter [3] to include the radial modes and later by Suzuki, Chin, and Satoh [4] to make an expansion in both azimuthal and radial modes.

Initially, the choice of the unperturbed distributions, for instance Gaussian, was made on the basis of making the equation easier to solve. It is therefore not a self-consistent approach for an electron bunch because it has its own equilibrium distribution. To improve the theory, Oide and Yokoya [5] studied the perturbation near Haissinski distribution [6]. They derived a generalized Sacherer integral equation that includes the incoherent tune shift due to the distorted potential. Moreover, they introduced the action-angle variables so that a Fourier expansion can be made in the angular variable to take the advantage of a periodicity in the system of bunched beam. For the radial direction, they numerically solved the integral equation by discretizing the action variable. Their method was successfully applied [7] to study the instability of the Stanford linear collider (SLC) damping ring, where many precision measurements were made [8]. However, it was found out later [9] that the method of discretization was not as robust as one might have expected largely due to the presence of

Published by the American Physical Society under the terms of the Creative Commons Attribution 3.0 License. Further distribution of this work must maintain attribution to the author(s) and the published article's title, journal citation, and DOI. the incoherent spectrum in the system. In particular, the convergence of the procedure is poor in many cases as one refines the mesh. To improve the theory, the integral equation was "regularized" by Warnock, Stupakov, Venturini, and Ellison [10] by replacing the linear equation with a highly nonlinear one, similar to the dispersion equation for a coasting beam. As demonstrated for the instability in the SLC damping ring, it also worked extremely well. However, the nonlinear equation itself introduced another layer of complication. Instead of solving an eigenvalue problem, one has to perform a search of a very nonlinear equation. In general, it is not clear if the method of search is indeed better than the one of Oide and Yokoya.

In this paper, we will continue the investigation of the stability of the Haissinski distribution by analyzing the linearized Vlasov (LV) equation. In particular, we will apply the orthogonal polynomials to solve the integral equation in the presence of the potential-well distortion.

We will start with the Vlasov-Fokker-Planck (VFP) equation in Sec. II; and then, in Sec. III, we will introduce the Haissinski distribution as a static solution of the VFP equation. In Sec. IV, we will derive the LV equation as a small perturbation near the Haissinski distribution. Then we will reduce the linear equation to an integral equation using a Fourier expansion in Sec. V. Finally, in Sec. VI, we will solve the integral equation in terms of various matrix representations.

In the latter part of the paper, we will apply the linear theory to commonly known impedances. First, the SLC damping ring will be studied as a benchmark in Sec. VII; second, we will continue, in Sec. VIII, to investigate the instability caused by the coherent synchrotron radiation (CSR) in free space; and third, we will study a broadband resonance model in Sec. VIII. At the end of the paper, we will conclude our study and discuss the microwave instability. 


\section{LONGITUDINAL BEAM DYNAMICS}

Let us consider an electron in a storage ring executing a small synchrotron oscillation in a stationary $\mathrm{rf}$ bucket. For simplicity, we introduce a normalized coordinate system, $q=z / \sigma_{z}$ and $p=-\delta / \sigma_{\delta}$, where $z$ is the differential position relative to the synchronized particle with energy $E_{0}, \delta=\left(E-E_{0}\right) / E_{0}$, and $\sigma_{z}$ and $\sigma_{\delta}$ are the standard deviations of position and relative energy in the equilibrium Gaussian distribution. Here we use positive $q$ as the forward direction of the beam. It is well known [11] that the bunch length $\sigma_{z}=\eta c \sigma_{\delta} / \omega_{s}$, where $\omega_{s}$ is the angular frequency of the synchrotron oscillation and $\eta$ is the momentum compaction factor. The motion of the electron is a simple harmonic oscillator described by the Hamiltonian, $H=\frac{1}{2}\left(q^{2}+p^{2}\right)$, along with independent variable, $\theta=\omega_{s} t$.

In general, the electron also experiences a collective force induced by the bunch distribution $\lambda(q, \theta)$. Using the notion of an integrated wakefield $W(q)$ [12] in a single turn, the dynamics can be described by a Hamiltonian

$H=\frac{1}{2}\left(q^{2}+p^{2}\right)-I_{n} \int_{-\infty}^{q} d q^{\prime \prime} \int_{-\infty}^{\infty} d q^{\prime} W\left(q^{\prime \prime}-q^{\prime}\right) \lambda\left(q^{\prime}, \theta\right)$,

where

$$
I_{n}=\frac{r_{e} N_{b}}{2 \pi \nu_{s} \gamma \sigma_{\delta}}
$$

is the normalized current, which was introduced by Oide and Yokoya [5]. $N_{b}$ represents the number of electrons in the bunch, $\nu_{s}$ is the synchrotron tune, $r_{e}$ is the classic radius of electron, and $\gamma=E_{0} / m c^{2}$. Here, the bunch distribution $\lambda(q, \theta)$ has been normalized, namely $\int_{-\infty}^{\infty} \lambda(q, \theta) d q=1$.

It is worth noting that the dynamics effect of the wakefield is scaled by the normalized current $I_{n}$, which is related to the bunch current $I_{b}$ through

$$
I_{b}=\eta \gamma \sigma_{\delta}^{2} I_{A}\left(\frac{I_{n}}{\sigma_{z}}\right)
$$

where $I_{A}=e c / r_{e}=17045 \mathrm{~A}$ is the Alfven current. It is clear from this formula that a higher energy, a larger momentum compaction factor, or a larger relative energy spread results in a higher threshold of the bunch current $I_{b}$ for a given threshold of the normalized current $I_{n}$. In this paper, we will develop a theory to calculate the threshold of $I_{n}$.

Furthermore, it can be shown that the evolution of the beam density distribution $\Psi(q, p ; \theta)$ is governed by the VFP equation

$$
\frac{\partial \Psi}{\partial \theta}-\{H, \Psi\}_{P B}=2 \beta \frac{\partial}{\partial p}\left(p \Psi+\frac{\partial \Psi}{\partial p}\right),
$$

where $\beta=1 / \omega_{s} \tau_{d}$ and $\tau_{d}$ is the longitudinal damping time. We use the subscript PB to indicate the Poisson bracket

$$
\{f, g\}_{\mathrm{PB}}=\frac{\partial f}{\partial q} \frac{\partial g}{\partial p}-\frac{\partial f}{\partial p} \frac{\partial g}{\partial q} .
$$

Actually, $H$ is the Hamiltonian defined in Eq. (2.1) with the substitution of $\lambda(q, \theta)=\int_{-\infty}^{\infty} \Psi(q, p ; \theta) d p$. As a result, the VFP equation is a nonlinear integral and partial differential equation. In general, it can only be solved by numerical methods [13]. In fact, it is a special form of the FokkerPlanck equation since the damping and diffusion terms on the right-hand side involve only the partial derivatives of $p$. This is a consequence of the fact that the synchrotron radiation causes loss and quantum diffusion only in the energy of the radiating electron not in its time of flight.

\section{HAISSINSKI DISTRIBUTION}

Historically, it was Haissinski who discovered that the VFP equation [Eq. (2.4)] has a static solution in the form of [6]

$$
\Psi_{0}(q, p)=\frac{1}{\kappa \sqrt{2 \pi}} \exp \left(-H_{0}\right)=\lambda_{0}(q) \exp \left(-\frac{p^{2}}{2}\right) / \sqrt{2 \pi},
$$

where $\kappa$ is a constant determined by the condition, $\int_{-\infty}^{\infty} \lambda_{0}(q) d q=1$. Here the subscript " 0 " indicates that the solution does not explicitly depend on $\theta$ or $\partial \Psi / \partial \theta=0$. Since $\Psi_{0}$ is a function of Hamiltonian $H_{0}$ only, it commutes with $H_{0}$ in the Poisson bracket and therefore the right-hand side of the equation vanishes by itself.

On the other hand, $\Psi_{0}$ is also factorized into a product of a Gaussian distribution in $p$ and $\lambda_{0}(q)$, which makes the right-hand side of the equation vanish separately. Using Eq. (2.1) for $H_{0}$ and eliminating the dependence of $p$ in Eq. (3.1), we find the well-known Haissinski integral equation:

$$
\begin{aligned}
\lambda_{0}(q)= & \frac{1}{\kappa} \exp \left[-\frac{q^{2}}{2}+I_{n} \int_{-\infty}^{q} d q^{\prime \prime}\right. \\
& \left.\times \int_{-\infty}^{\infty} d q^{\prime} W\left(q^{\prime \prime}-q^{\prime}\right) \lambda_{0}\left(q^{\prime}\right)\right] .
\end{aligned}
$$

At zero current, $I_{n}=0$, so the solution becomes a Gaussian. In general, this nonlinear integral equation can be solved numerically using Newton iteration starting from the Gaussian distribution. A Haissinski solution, for the SLC damping ring, is shown in Fig. 1.

In practice, we know that the Haissinski distribution is not just a possible solution but also the equilibrium distribution of the VFP equation at a sufficiently low current. Above a threshold of $I_{n}$, the Haissinski distribution is no longer a stable solution. In the literature, the associated instability is commonly referred to as the microwave instability. 


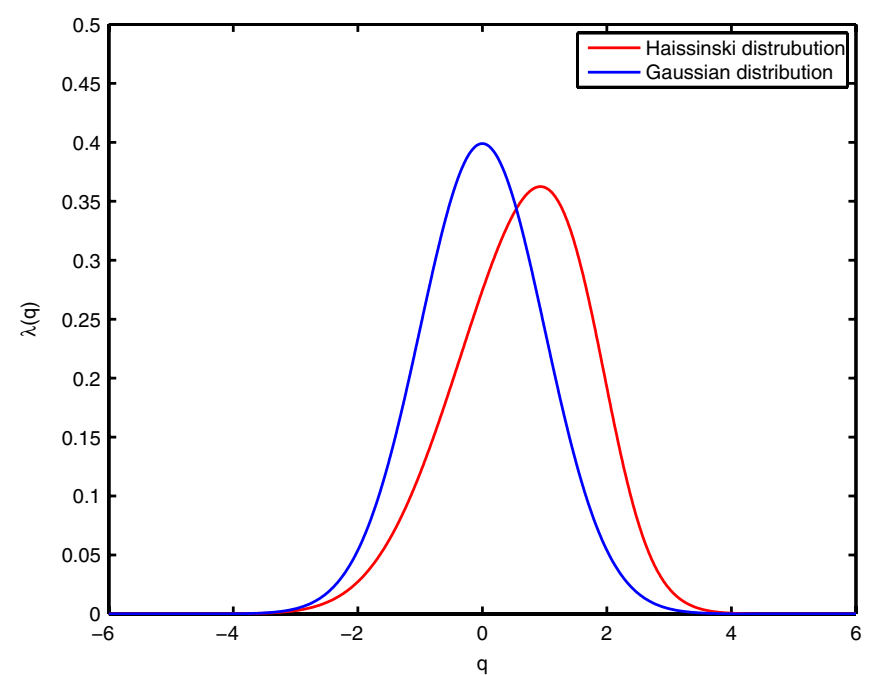

FIG. 1. Comparison of Haissinski distribution at the threshold current $I_{n}^{\text {th }}=0.042 \mathrm{pC} / \mathrm{V}$ in the SLC damping ring to a Gaussian distribution.

\section{LINEARIZED VLASOV EQUATION}

At the limit of $\beta$ going to zero, the VFP equation reduces to the Vlasov equation:

$$
\frac{\partial \Psi}{\partial \theta}-\{H, \Psi\}_{\mathrm{PB}}=0 .
$$

For an estimate of the threshold of instability, one often uses the Vlasov equation because the damping time is much longer than the synchrotron period in a typical electron storage ring and, hence, $\beta$ is a small and dimensionless parameter. For instance, $\beta=0.00091$ for the SLC damping ring.

To investigate the instability, we expand the density distribution:

$$
\Psi(\phi, J ; \theta)=\Psi_{0}\left(H_{0}\right)+\Psi_{1}(\phi, J ; \theta),
$$

where $\Psi_{1}(\phi, J ; \theta)$ is a small perturbation away from the Haissinski distribution $\Psi_{0}\left(H_{0}\right)$. Here we choose to use the action-angle variables, $J$ and $\phi$, which can be canonically transformed from $q$ and $p$. Substituting the expansion into the Vlasov equation [Eq. (4.1)] and ignoring the nonlinear terms in $\Psi_{1}$, we obtain a $L V$ equation,

$$
\frac{\partial \Psi_{1}}{\partial \theta}+\left(\frac{\partial \Psi_{1}}{\partial \phi}-\frac{\partial H_{1}}{\partial \phi} \frac{d \Psi_{0}}{d H_{0}}\right) \frac{\omega(J)}{\omega_{s}}=0,
$$

where $\omega(J) / \omega_{s} \equiv d H_{0} / d J$ and $H_{1}$ is the perturbed Hamiltonian.

$H_{1}=-I_{n} \int_{-\infty}^{q} d q^{\prime \prime} \int_{0}^{2 \pi} d \phi^{\prime} \int_{0}^{\infty} d J^{\prime} W\left(q^{\prime \prime}-q^{\prime}\right) \Psi_{1}\left(\phi^{\prime}, J^{\prime} ; \theta\right)$.

We have used the fact that the Poisson bracket is invariant and $d q d p=d \phi d J$ under a canonical transformation.

\section{SACHERER INTEGRAL EQUATION}

Following Sacherer's approach [1], we make a Fourier expansion of $\Psi_{1}$ in $\phi$ :

$$
\Psi_{1}(\phi, J ; \theta)=\sum_{l=-\infty}^{\infty} R_{l}(J) e^{i l \phi} e^{-i\left(\Omega / \omega_{s}\right) \theta} .
$$

Substituting this expansion into the LV equation and using the orthogonal condition of a Fourier transformation, we derive the generalized Sacherer integral equation,

$$
\begin{aligned}
& \left(\frac{\Omega}{\omega(J)}-l\right) R_{l}(J) \\
& \quad=W\left(H_{0}\right) \sum_{m=-\infty}^{\infty} \int_{0}^{\infty} d J^{\prime} G_{l, m}\left(J, J^{\prime}\right) R_{m}\left(J^{\prime}\right)
\end{aligned}
$$

where the kernel is

$G_{l, m}\left(J, J^{\prime}\right)=\frac{i I_{n}}{2 \pi} \int_{0}^{2 \pi} d \phi e^{-i l \phi} \int_{0}^{2 \pi} d \phi^{\prime} e^{i m \phi^{\prime}} W\left(q-q^{\prime}\right) \frac{\partial q}{\partial \phi}$,

and $W\left(H_{0}\right)=-d \Psi_{0}\left(H_{0}\right) / d H_{0}$. This integral equation was studied by Oide and Yokoya [5]. Actually, as one can see in the Appendix, it is more convenient to use the free energy $K=H_{0}-V_{\min }$ as the radial variable. The static nonzero value $V_{\min }$ is subtracted to make the minimum value of $K$ be zero. The transformation between $q, p$ and $\phi, K$ will be given in the Appendix. Using $K$ to replace $J$, the Sacherer integral equation becomes

$$
\begin{aligned}
\left(\frac{\Omega}{\omega_{s}}\right. & \left.-l \frac{\omega(K)}{\omega_{s}}\right) P_{l}(K) \\
& =\frac{e^{-V_{\min }}}{\kappa \sqrt{2 \pi}} e^{-K} \sum_{m=-\infty}^{\infty} \int_{0}^{\infty} d K^{\prime} G_{l, m}\left(K, K^{\prime}\right) P_{m}\left(K^{\prime}\right),
\end{aligned}
$$

where $P_{l}(K)=\omega_{s} R_{l}(K) / \omega(K)$. Here we have used Eq. (3.1) for $\Psi_{0}$.

Moreover, we can introduce the impedance $Z(\omega)$ to replace the wakefield:

$$
W\left(q-q^{\prime}\right)=\frac{1}{2 \pi} \int_{-\infty}^{\infty} d \omega e^{i\left(\omega \sigma_{z} / c\right)\left(q-q^{\prime}\right)} Z(\omega),
$$

in the kernel $G_{l, m}\left(K, K^{\prime}\right)$ in Eq. (5.3). By performing an integration by parts for $q$ and using the periodical condition, $q(\phi+2 \pi, K)=q(\phi, K)$, the kernel can be simplified to be

$$
\begin{aligned}
& G_{l, m}\left(K, K^{\prime}\right) \\
& \quad=\left(\frac{i I_{n} c l}{\sigma_{z}}\right) \int_{-\infty}^{\infty} d \omega \frac{Z(\omega)}{\omega} h_{l}\left(\frac{\omega \sigma_{z}}{c}, K\right) h_{m}^{*}\left(\frac{\omega \sigma_{z}}{c}, K^{\prime}\right),
\end{aligned}
$$

where

$$
h_{l}(\nu, K)=\int_{0}^{2 \pi} \frac{d \phi}{2 \pi} e^{-i l \phi+i \nu q(\phi, K)}
$$


Furthermore, using the symmetry $q(-\phi, K)=q(\phi, K)$ and the property of impedance $Z(-\omega)=Z(\omega)^{*}$, we obtain

$$
\begin{aligned}
G_{l, m}\left(K, K^{\prime}\right)= & \frac{-l I_{n} c}{\sigma_{z}} \operatorname{Im}\left[2 \int_{0}^{\infty} d \omega \frac{Z(\omega)}{\omega}\right. \\
& \left.\times h_{l}\left(\frac{\omega \sigma_{z}}{c}, K\right) h_{m}^{*}\left(\frac{\omega \sigma_{z}}{c}, K^{\prime}\right)\right] .
\end{aligned}
$$

Clearly, the kernel itself is a real not a complex function.

\section{SOLVE THE INTEGRAL EQUATION}

We would like to solve $P_{l}(K)$ along with $\Omega$ in Eq. (5.4). According to Eq. (5.1), the real part of $\Omega$ contributes to merely a coherent tune shift and its positive imaginary part causes an exponential growth of the perturbation $\Psi_{1}$. The goal is to find the threshold of current at which $\Omega$ develops a positive imaginary value.

\section{A. Orthogonal polynomial expansion}

A method of solving the integral equation is to use orthogonal polynomials [2]. We further decompose

$$
P_{l}(K)=e^{-K} \sum_{\alpha=0}^{\infty} a_{l}^{\alpha} f_{\alpha}^{(l)}(K),
$$

where $f_{\alpha}^{(l)}$ are polynomials that satisfy the orthogonal and normal conditions:

$$
\int_{0}^{\infty} d K e^{-K} f_{\alpha}^{(l)}(K) f_{\beta}^{(l)}(K)=\delta_{\alpha \beta} .
$$

A similar expansion of the radial modes were used by Zotter [3], Suzuki, Chin, and Satoh [4] when they studied a Gaussian model. An obvious difference from theirs is that our variable $K$ is the free energy rather than the radius. Most importantly, the incoherent tune variation $\omega(K)$ is a weighting factor in the radial expansion of $R_{l}(K)$.

Using the orthogonal condition of the polynomials, we can cast the integral equation [Eq. (5.4)] into an infinite system of linear equations:

$$
\frac{\Omega}{\omega_{s}} a_{\alpha}^{l}=\sum_{m=-\infty}^{\infty} \sum_{\beta=0}^{\infty} M_{l, m}^{\alpha, \beta} a_{\beta}^{m} .
$$

It is clear that the eigenvalues of the matrix $M$ give us $\Omega / \omega_{s}$ since this equation itself defines eigenvalues. In this approach, we need to solve the eigenequations.

Actually, the matrix $M=O+N$ is the sum of two matrices. The element of one of the matrices is given by

$$
O_{l, m}^{\alpha, \beta}=l \delta_{l m} \int_{0}^{\infty} d K \frac{\omega(K)}{\omega_{s}} e^{-K} f_{\alpha}^{(l)}(K) f_{\beta}^{(l)}(K),
$$

which describes a direct coupling of the radial modes with the same azimuthal mode number due to the incoherent tune shift $\omega(K)$ generated from the potential-well distortion.
The other matrix element $N_{l, m}^{\alpha, \beta}$ is written as

$$
\begin{aligned}
N_{l, m}^{\alpha, \beta}= & \frac{e^{-V_{\min }}}{\kappa \sqrt{2 \pi}} \int_{0}^{\infty} d K e^{-K} f_{\alpha}^{(l)}(K) \\
& \times \int_{0}^{\infty} d K^{\prime} e^{-K^{\prime}} f_{\beta}^{(m)}\left(K^{\prime}\right) G_{l, m}\left(K, K^{\prime}\right) .
\end{aligned}
$$

Furthermore, the polynomials $f_{\alpha}^{(l)}(K)$ can be easily constructed from the generalized Laguerre polynomials $L_{\alpha}^{|l|}(K)$, namely,

$$
f_{\alpha}^{(l)}(K)=\sqrt{\frac{\alpha !}{(|l|+\alpha) !}} K^{|l| / 2} L_{\alpha}^{|l|}(K) .
$$

Here we use only the absolute value of the azimuthal mode number $l$ in the expression so that both positive and negative $l$ are treated equally in the expansion $P_{l}(K)$.

Using Eq. (5.8) for the kernel $G_{l, m}\left(K, K^{\prime}\right)$, we obtain

$$
\begin{aligned}
N_{l, m}^{\alpha, \beta}= & \frac{-l I_{n} c e^{-V_{\min }}}{\kappa \sigma_{z} \sqrt{2 \pi}} \operatorname{Im}\left[2 \int_{0}^{\infty} d \omega \frac{Z(\omega)}{\omega}\right. \\
& \left.\times g_{l}^{\alpha}\left(\frac{\omega \sigma_{z}}{c}\right) g_{m}^{\beta^{*}}\left(\frac{\omega \sigma_{z}}{c}\right)\right],
\end{aligned}
$$

where

$$
g_{l}^{\alpha}(\nu)=\int_{0}^{\infty} d K e^{-K} f_{\alpha}^{(l)}(K) h_{l}(\nu, K) .
$$

It is worth noting that the dependence of $N_{l, m}^{\alpha, \beta}$ on the current $I_{n}$ is not linear as the expression indicates, since both $V_{\min }$ and $\kappa$ implicitly depend on $I_{n}$ through the nonlinear Haissinski equation. That is one of the major differences between the self-consistent treatment and a Gaussian model.

For a Gaussian model, we have a potential of a harmonic oscillator, $V(q)=q^{2} / 2$, which results in $\kappa=\sqrt{2 \pi}$ and $V_{\min }=0$. Substituting them into Eqs. (A4) and (A5) and evaluating the integrals, we find $q=\sqrt{2 K} \cos \phi$ and $\omega(K) / \omega_{s}=1$. These simple analytical expressions allow us to further evaluate $O_{l, m}^{\alpha, \beta}$ and $N_{l, m}^{\alpha, \beta}$. After some straightforward calculations, we obtain $O_{l, m}^{\alpha, \beta}=l \delta_{l m} \delta_{\alpha \beta}$,

$$
\begin{aligned}
h_{l}^{G}(\nu, K) & =i^{l} J_{l}(\nu \sqrt{2 K}), \\
g_{l}^{\alpha G}(\nu) & =i^{l}\left(\frac{\nu}{\sqrt{2}}\right)^{|l|+2 \alpha} \sqrt{\frac{1}{\alpha !(|l|+\alpha) !}} e^{-\nu^{2} / 2},
\end{aligned}
$$

and

$$
\begin{aligned}
N_{l, m}^{\alpha, \beta}= & \frac{i c I_{n}}{2 \pi \sigma_{z}}\left(\frac{l i^{l-m}}{\sqrt{\alpha !(|l|+\alpha) ! \beta !(|m|+\beta) !}}\right) \\
& \times \int_{-\infty}^{\infty} d \omega \frac{Z(\omega)}{\omega} e^{-\left(\omega \sigma_{z} / c\right)^{2}}\left(\frac{\omega \sigma_{z}}{\sqrt{2} c}\right)^{|l|+|m|+2(\alpha+\beta)} .
\end{aligned}
$$


These matrix elements agree with those derived by Suzuki, Chin, and Satoh [4] in their Gaussian model. Clearly, our result contains the Gaussian beam as a special case.

\section{B. Oide and Yokoya discretization}

Another method to solve the integral equation is to discretize the variable $K$ from 0 to $K_{\max }$ and to approximate the integration with a summation. This method leads to the same eigenequation as Eq. (6.3) with the matrix elements

$$
O_{l, m}^{\alpha, \beta}=\frac{l \omega\left(K_{\alpha}\right)}{\omega_{s}} \delta_{l m} \delta_{\alpha \beta}
$$

and

$$
N_{l, m}^{\alpha, \beta}=\frac{\Delta K e^{-V_{\min }-K_{\alpha}}}{\kappa \sqrt{2 \pi}} G_{l, m}\left(K_{\alpha}, K_{\beta}\right),
$$

where $K_{\alpha}=\alpha \Delta K$ are the grid points and $\Delta K$ is the spacing of the grid.

In practice, both methods of polynomial expansion and discretization are merely an approximation since the expansion has to be truncated either by the highest order of the polynomials or the largest number of grid points on the radial mesh. With the polynomial expansion, one has to carry out two more integrals to obtain the matrix elements in exchange for a smaller dimension of the matrix. In general, it is not clear which method is more efficient to achieve the necessary convergence. In the following sections, we will study the instability caused by some commonly known impedances as examples.

\section{SLC DAMPING RING}

The longitudinal instabilities in the SLC damping rings were well studied both experimentally [8] and theoretically $[7,10]$. Naturally, it serves as an excellent example for a benchmark. As one will see later, it is also a simple system in which only a single quadupole-like mode is excited in a large range of current above the threshold.

For the SLC damping ring, its longitudinal wakefield and impedance were carefully calculated by Bane and $\mathrm{Ng}$ [14]. Because of a high periodicity in the ring, an accurate model, as shown in Fig. 2, was possible to be constructed with a reasonable effort. As one can see in the impedance plot on the right, the model may be approximated by a constant resistance and a pure inductance.

For a given current $I_{n}$, we first solve the Haissinski integral equation for a solution of $\lambda_{0}(q)$ on a rectangular mesh of $q$ and $p$ using the wakefield. As a by-product of the equation solving, we can obtain the distorted potential well $V(q)$. Second, we use the transformation to compute $\omega(K)$ and $q=q(\phi, K)$ as illustrated in the Appendix and save the results as a numerical table. Third, we perform numerical integration of $\phi$ for $h_{l}(\nu, K)$. As an example, we show $h_{2}(\nu, 2.0)$ on the left in Fig. 3. For the Oide and Yokoya method, we can compute the kernel by integrating the frequency $\omega$ using Eq. (5.8) and the matrix elements by Eqs. (6.11) and (6.12).

For the method of Laguerre polynomials, we need to perform integrals of $K$ from zero to infinity. Since the functions of $\omega(K)$ and $h_{l}(\nu, K)$ are defined only in a finite range $0<K<K_{\max }$, we extrapolate them into the region beyond $K_{\max }$ using the Gaussian model. This approximation is justified because most potentials have the same asymptote as the potential of a linear oscillator $V(q)=$ $q^{2} / 2$ when $q$ approaches to infinity. One may argue that the linear oscillator itself is valid only for a small $q$. Here we ignore the effects such as the nonlinear if potential. For the elements of matrix $O$, we have
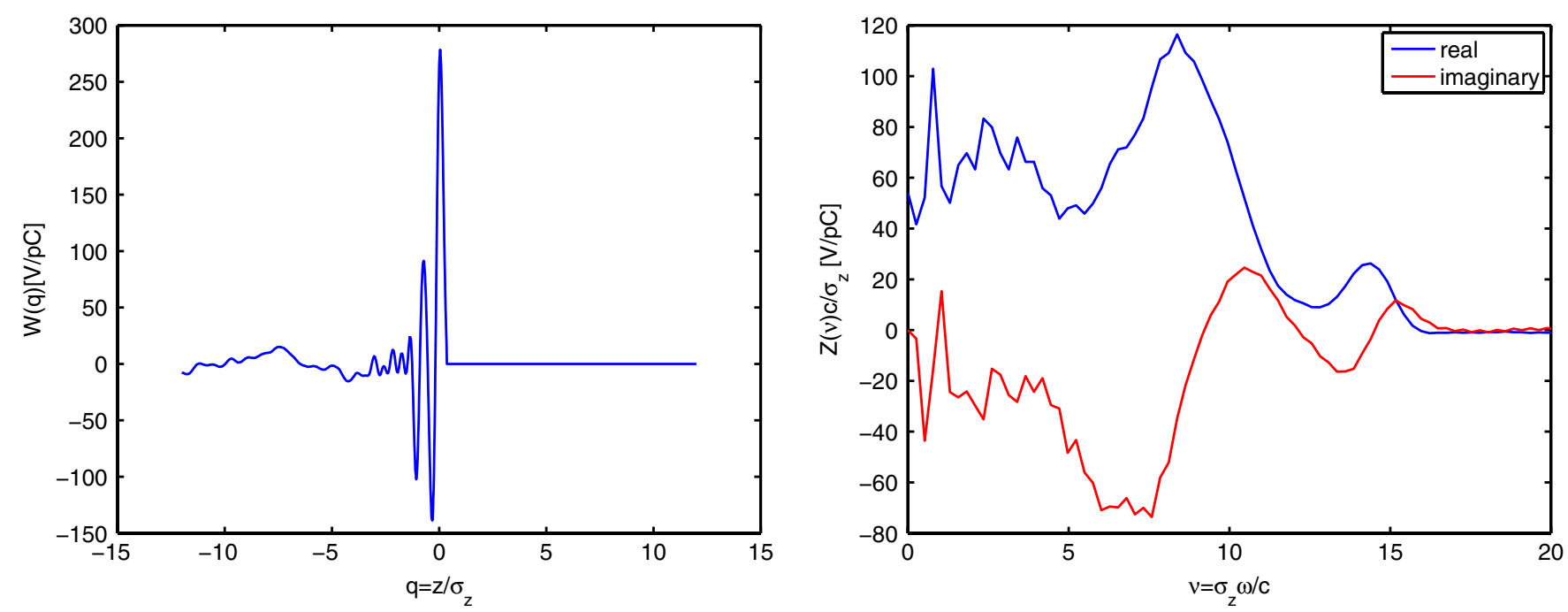

FIG. 2. Wakefield (left) and impedance (right) for the SLC damping ring for $\sigma_{z}=4.946 \mathrm{~mm}$ (bunch length at zero current). 

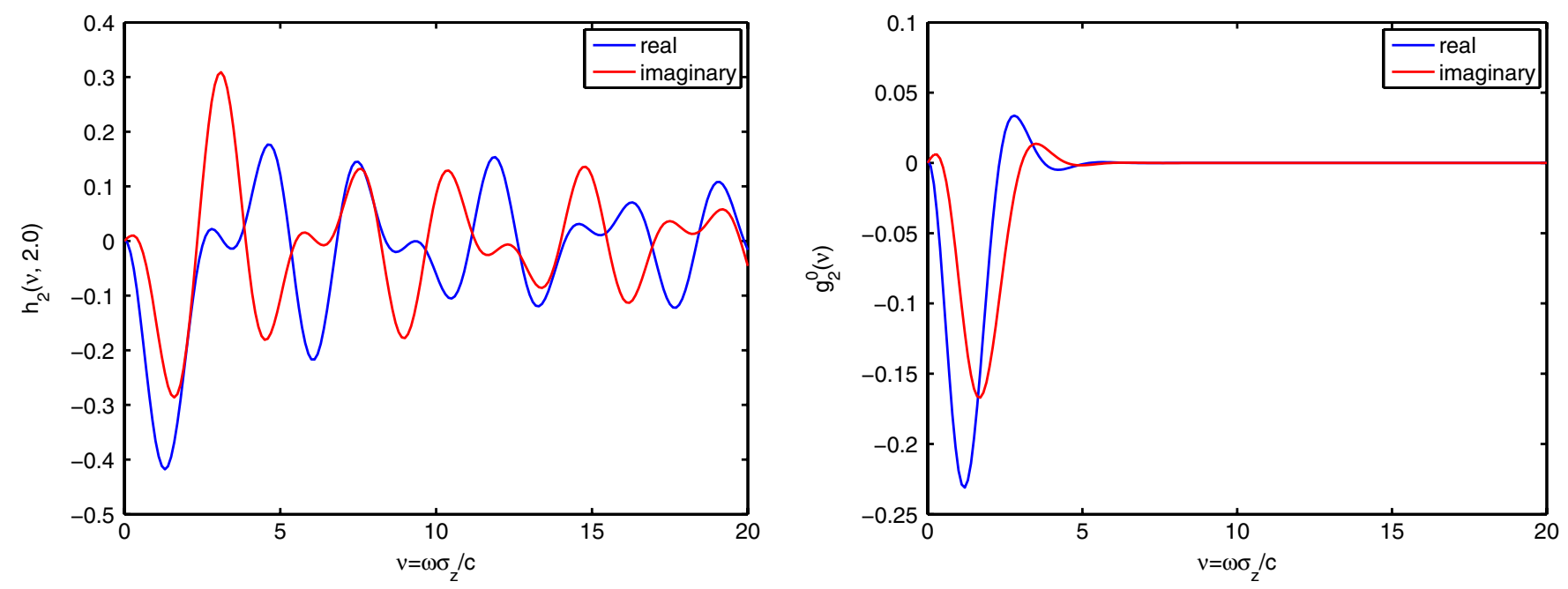

FIG. 3. Spectrum functions at the threshold current $I_{n}^{\text {th }}=0.042 \mathrm{pC} / \mathrm{V}$ in the SLC damping ring. For the Gaussian model, their imaginary parts should be vanishing for the $l=2$ mode.

$$
\begin{aligned}
O_{l, m}^{\alpha, \beta}= & l \delta_{l m} \int_{0}^{K_{\max }} d K\left[\frac{\omega(K)}{\omega_{s}}-1\right] e^{-K} f_{\alpha}^{(l)}(K) f_{\beta}^{(l)}(K) \\
& +l \delta_{l m} \delta_{\alpha \beta},
\end{aligned}
$$

and similarly

$$
g_{l}^{\alpha}(\nu)=\int_{0}^{K_{\max }} d K e^{-K} f_{\alpha}^{(l)}(K)\left[h_{l}(\nu, K)-h_{l}^{G}(\nu, K)\right]+g_{l}^{\alpha G}(\nu) .
$$

As an example, we show $g_{2}^{0}(\nu)$ on the right in Fig. 3. As one can see from the figure, the large value of the function is near $\nu=2$ and damped down quickly at high frequency. As a result, the high frequency impedance does not contribute to the quadrupole mode. Finally, we compute $N_{l, m}^{\alpha, \beta}$ by integrating $\omega$ in Eq. (6.7). With the elements of the

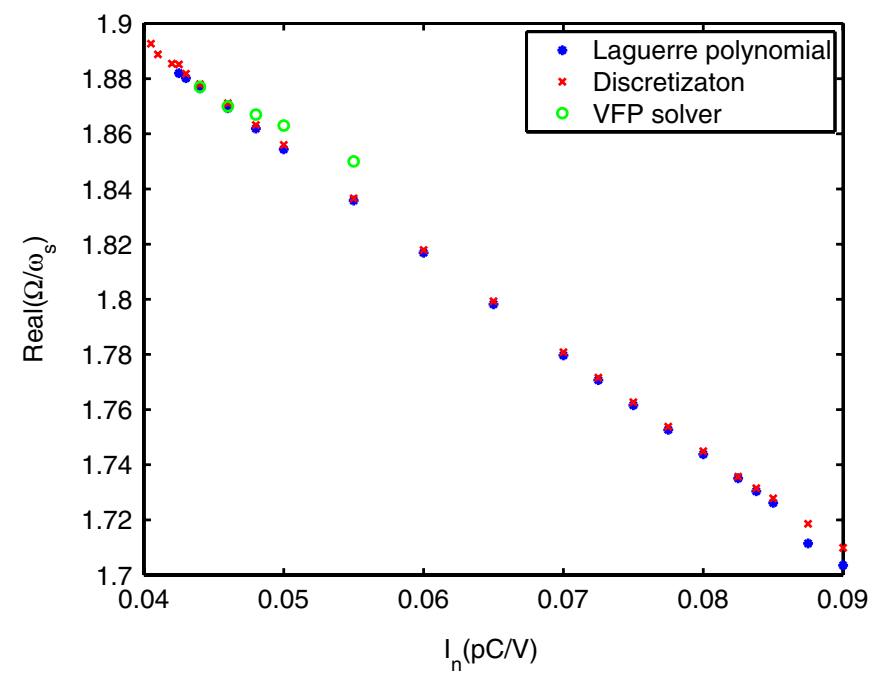

matrix $M=O+N$, we numerically calculate its eigenvalues and eigenfunctions.

This procedure is repeated for many different values of $I_{n}$, starting from zero. At low currents, all eigenvalues are real and the bunch is stable. The instability threshold is defined as the current at which the first imaginary eigenvalue is found as the current increases. The results of our analysis are summarized and shown in Fig. 4. The most unstable mode is a quadrupole-like mode. As one can see, the agreement between the results obtained by three different methods is excellent. We used ten azimuthal modes in our calculations. For the method of discretization, we used $\Delta K=0.2$ (approximately 80 grid points) in the radial direction. For the method of Laguerre polynomials, we used the integration step $d K=0.01$ and 30 radial modes in the expansion. Here, we essentially reproduced the wellknown results from the earlier works by Bane and Oide [7]

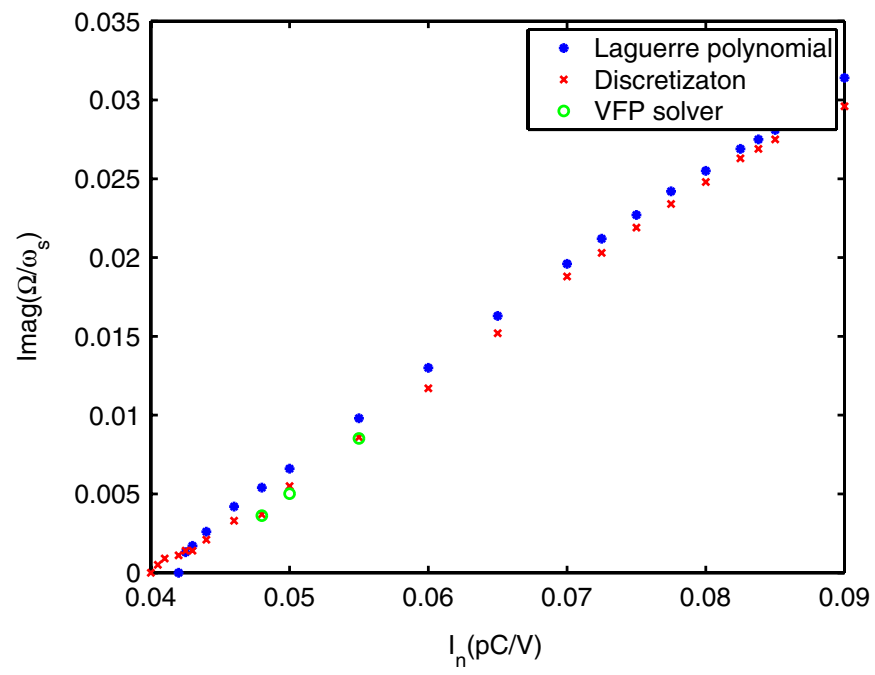

FIG. 4. Tune shift (left) and growth rate (right) of the most unstable mode in the SLC damping ring. 
and later by Warnock, Stupakov, Venturini, and Ellison [10]. We expect to see a good agreement between the calculations since both procedures converged rather well.

The VFP simulations were carried out using the code [13] provided to us by Warnock. The tune shifts were extracted from the Fourier analysis of the center of the bunch distribution $\langle q\rangle$; and the growth rate was obtained by fitting to the exponential increase of $\sigma_{p}$ and then subtracting out the radiation damping $2 \beta=0.0018$. In fact, this subtraction is corrected, according to a paper by Suzuki [15], only because the unstable mode is a quadrupole. A small deviation at higher currents can be attributed to the nonlinear effects in the VFP simulation.

Finally, a sextupole-like mode pops up at $I_{n}=$ $0.075 \mathrm{pC} / \mathrm{V}$ with its real part of $\Omega / \omega_{s}=2.59$ in the linear theory. It becomes the dominating mode at $I_{n}=$ $0.0825 \mathrm{pC} / \mathrm{V}$ with $\operatorname{Re}\left[\Omega / \omega_{s}\right]=2.55$. This result is consistent with the sextupole mode observed in the experiments [8]. The unexpected agreement surprised us since it occurs well beyond the threshold $I_{n}^{\text {th }}=0.042 \mathrm{pC} / \mathrm{V}$. But it is not clear why the sextupole mode is never seen in the VFP simulations.

\section{COHERENT SYNCHROTRON RADIATION}

As we have shown in the previous section, there is essentially a single quadrupole mode excited in a large range of beam current in the SLC damping ring. As a result, the linear theory works extremely well. In this section, we will study the instability due to CSR generated by circulating electrons inside bending magnets. This will provide another example to illustrate some features in our analysis.

Consider an electron executing circular motion with radius $\rho$ inside bending magnets. The longitudinal wakefield due to the steady CSR generated by another electron behind is given by [16]

$$
W(q)=\frac{-4 \pi \rho^{1 / 3}}{3^{4 / 3} \sigma_{z}^{4 / 3}} q^{-4 / 3} .
$$

In contrast to many other properties of synchrotron radiation, this wake does not depend on the energy of the electrons. As a result, it is universal to many types of electron storage rings. In particular, it can become a dominate collective effect as the bunch length $\sigma_{z}$ reduces down to the millimeter region. As one can easily see from the formula of the wake and the Vlasov equation [Eq. (4.1)], the theory depends only on a dimensionless parameter, namely $\xi=I_{n} \rho^{1 / 3} / \sigma_{z}^{4 / 3}$. [17]

The impedance is also well known and can be written

$$
Z(\omega)=\left(\frac{4 \pi}{c}\right)\left(\frac{\Gamma\left(\frac{2}{3}\right)}{3^{1 / 3}}\right)\left(\frac{\sqrt{3}}{2}+\frac{i}{2}\right)\left(\frac{\rho \omega}{c}\right)^{1 / 3}
$$

Substituting the impedance into Eq. (5.8), we obtain the kernel

$$
\begin{aligned}
G_{l, m}\left(K, K^{\prime}\right)= & \frac{-4 \pi \Gamma\left(\frac{2}{3}\right) l \xi}{3^{1 / 3}} \operatorname{Im}[(\sqrt{3}+i) \\
& \left.\times \int_{0}^{\infty} d \nu \nu^{-2 / 3} h_{l}(\nu, K) h_{m}^{*}\left(\nu, K^{\prime}\right)\right] .
\end{aligned}
$$

Similarly,

$$
\begin{aligned}
N_{l, m}^{\alpha, \beta}= & \frac{-2 \sqrt{2 \pi} e^{-V_{\min }} \Gamma\left(\frac{2}{3}\right) l \xi}{3^{1 / 3} \kappa} \operatorname{Im}[(\sqrt{3}+i) \\
& \left.\times \int_{0}^{\infty} d \nu \nu^{-2 / 3} g_{l}^{\alpha}(\nu) g_{m}^{\beta^{*}}(\nu)\right],
\end{aligned}
$$

using Eq. (6.7).

\section{A. Gaussian model}

For the Gaussian model, using $g_{l}^{\alpha G}(\nu)$ and carrying out the integral of $\nu$ in Eq. (8.4), we obtain

$$
\begin{aligned}
N_{l, m}^{\alpha, \beta}= & \frac{-\Gamma\left(\frac{2}{3}\right) l \xi}{3^{1 / 3} 2^{n / 2} \sqrt{(|l|+\alpha) ! \alpha !(|m|+\beta) ! \beta !}} \\
& \times \Gamma\left[\frac{1}{2}\left(n+\frac{1}{3}\right)\right] \operatorname{Im}\left[(\sqrt{3}+i) i^{l-m}\right],
\end{aligned}
$$

where $n=|l|+|m|+2 \alpha+2 \beta$. We use these elements along with $O_{l, m}^{\alpha, \beta}=l \delta_{l m} \delta_{\alpha \beta}$ to compute the eigenvalues of the matrix $M=O+N$. Our result gives a classic picture of mode coupling as shown in Fig. 5.

At the threshold of $\xi^{\text {th }}=0.578$, we see the first merge of $l=1$ and $l=2$ modes. The merge of the modes generates a pair of complex modes with equally positive and negative imaginary values. Here we plot only the positive ones. Ten radial modes and 50 azimuthal modes are used in the

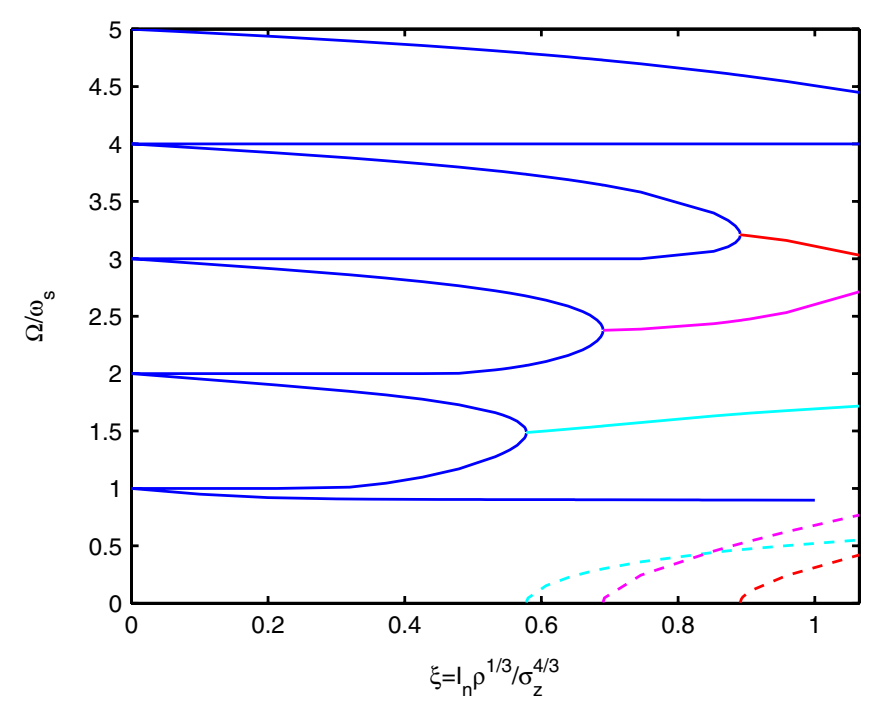

FIG. 5. Mode analysis using the Gaussian model. Solid lines represent the coherent tune shifts $\left[\operatorname{Re}\left(\Omega / \omega_{s}\right)\right]$ and dashed lines are for the growth rates $\left[\operatorname{Im}\left(\Omega / \omega_{s}\right)\right]$ of the unstable modes. For a given azimuthal mode number, only the radial modes with the largest and smallest tune shifts are shown. 
calculation. There is no problem to achieve $0.1 \%$ level of convergence to determine the threshold.

\section{B. Self-consistent methods}

For a self-consistent treatment, we need to solve the Haissinski and Sacherer integral equations at each current; then we repeat the procedure, outlined in the section for studying the SLC damping ring, for various currents. The expressions for kernel in Eq. (8.3) and for matrix elements in Eq. (8.4) simplify the calculation.

Using the expansion of Laguerre polynomials, we find the threshold at $\xi^{\text {th }}=0.482$ as illustrated in Fig. 6. In contrast to the instability of the SLC damping ring, many modes are simultaneously excited slightly above the threshold. Furthermore, as one can see from the left plot in the figure, an unstable azimuthal mode is as high as the 15th. To accommodate the high-order modes, we have used 20 azimuthal and 20 radial modes in our calculation. As shown in Fig. 7, a reasonably good convergence is achieved with 20 radial modes while the number of azimuthal modes is fixed at 20.

An obvious difference from the Gaussian model is that the threshold is lower by $20 \%$. A little subtle one is that there are more unstable modes, resulting in a slower growth rate for each mode. Finally, as we show in Fig. 8, the coupling modes are in the radial direction. As one can see from the figure, the first unstable mode is not a quadrupole mode but a sextupole mode. The threshold for the unstable quadrupole mode is slightly higher at $\xi_{q}^{\text {th }}=0.50$. This threshold agrees perfectly with the result using the VFP solver in our previously published paper [18]. The agreement should not be considered merely a coincidence since we had used a quadratic variance $p>1$ as the criterion to determine the microwave threshold in the

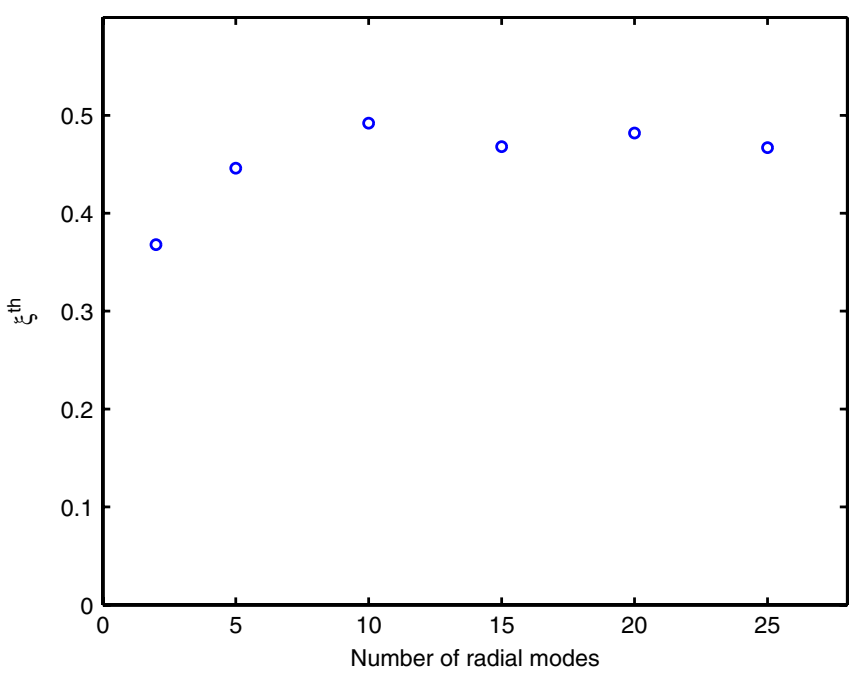

FIG. 7. Threshold of microwave instability as a function of number of radial modes expanding with the Laguerre polynomials.

simulation and therefore made it more sensitive for detecting the instability of a quadrupole mode.

As we discussed in the section on the SLC damping ring, we can also use the discretization to solve the Sacherer integral equation. However, as we discussed earlier, a much higher expansion of the azimuthal modes is required for the CSR impedance. As a result, the dimension of the matrix becomes so large, in terms of the use of computer memory, that the computation becomes too slow due to the swapping of memory. This limitation prevents us from achieving any good convergence. With the finest grid $\Delta K=0.1$ possible, the discretization method gives a much lower threshold at $\xi^{\text {th }}=0.25$ as shown in Fig. 9. Since the convergence is poor, it is not clear if the threshold
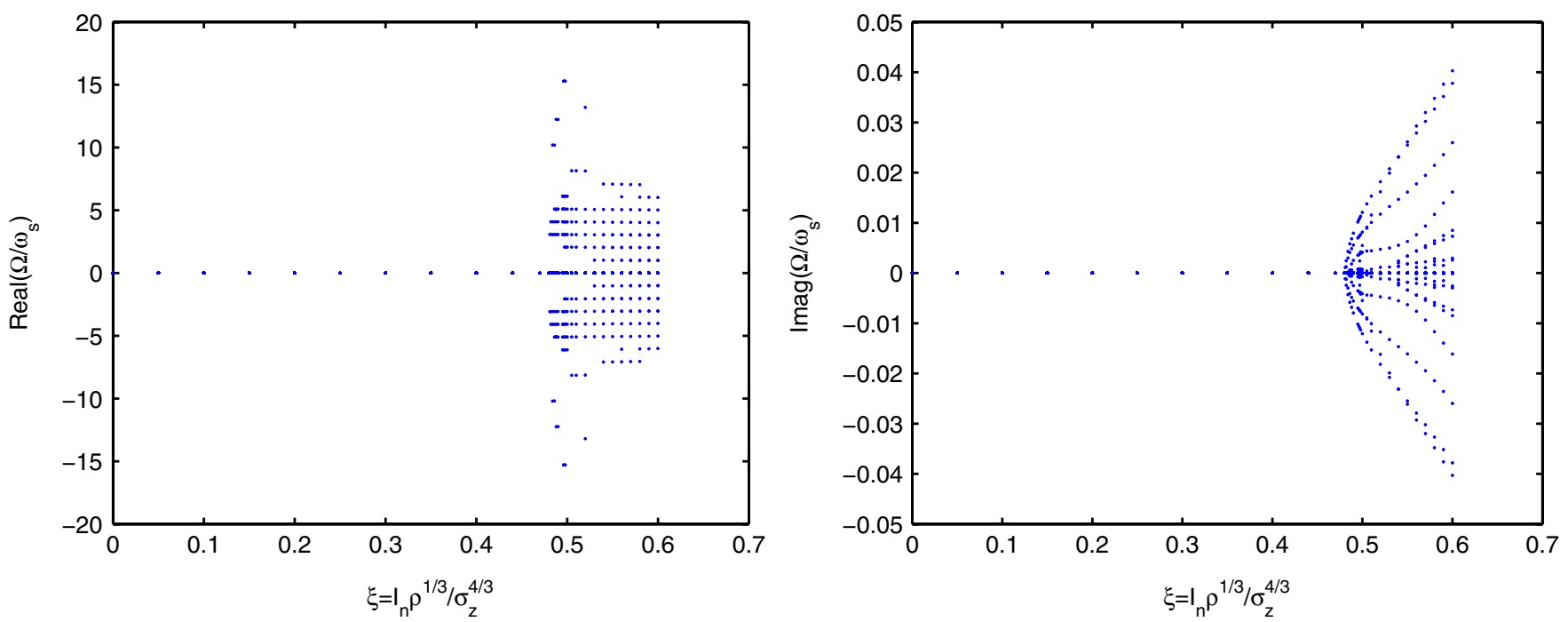

FIG. 6. Real parts (left) and imaginary parts (right) of all unstable modes driven by CSR in free space. Each dot represents an unstable mode. 

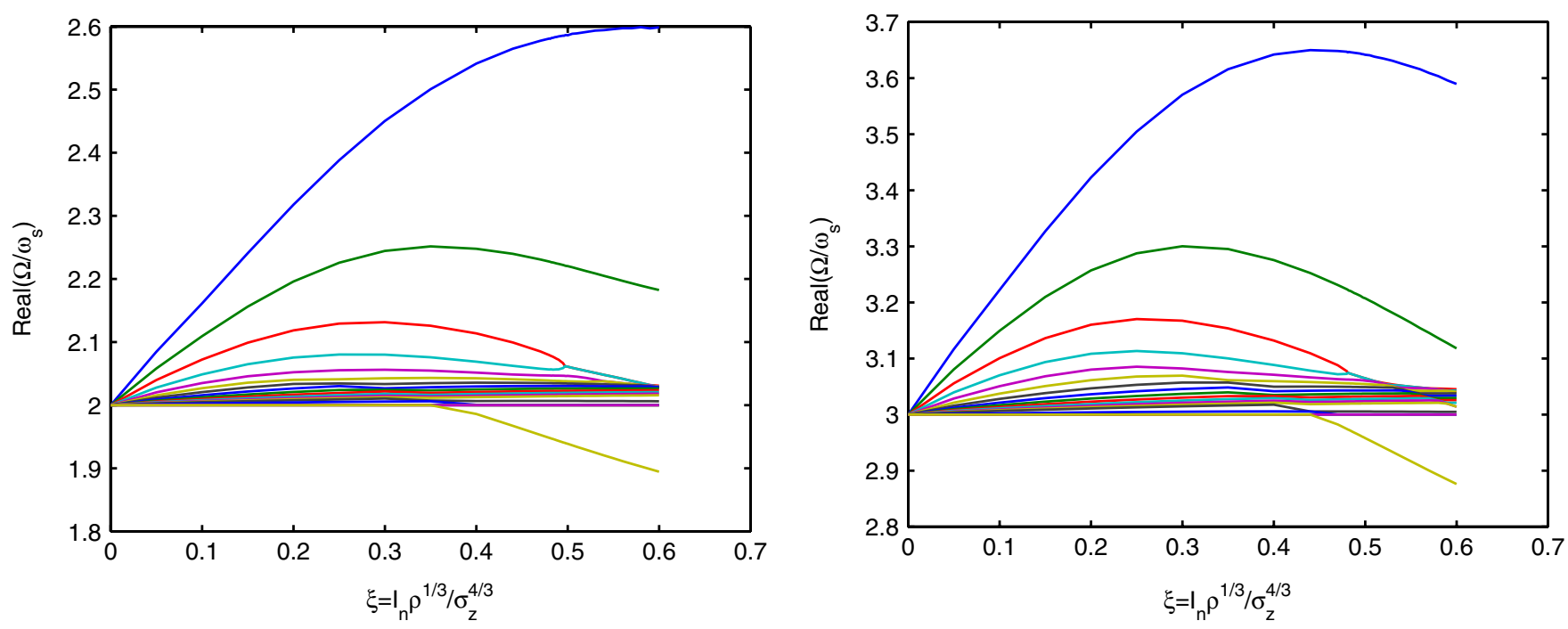

FIG. 8. Coupling of the radial modes: quadrupoles on the left and sextupoles on the right.

is indeed accurate. However, this lower threshold cannot be easily discarded because it is very close to the threshold calculated [19] by applying the theory of a coasting beam.

\section{BROADBAND RESONANCE MODEL}

Another commonly studied impedance model is a broadband resonance model constructed with a parallel circuit of an inductance, a resistance, and a capacitance [12],

$$
Z(\omega)=\frac{R}{1+i Q\left(\frac{\omega_{r}}{\omega}-\frac{\omega}{\omega_{r}}\right)},
$$

where $R$ is the resistance, $Q=R \sqrt{C / L}$ is the quality factor, and $\omega_{r}=1 / \sqrt{C L}$ is the resonance frequency. The wakefield can be calculated using Eq. (5.5). For $q<0$, it is given by

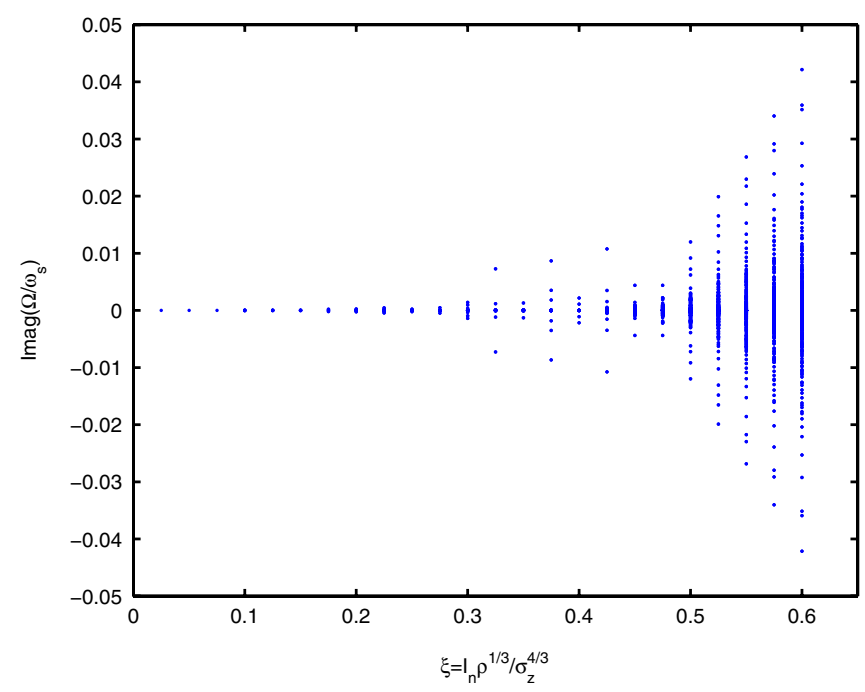

FIG. 9. Growth rates of all modes at various currents using the discretization method for the CSR impedance in free space.

$$
\begin{aligned}
W(q)= & \frac{R \omega_{r}}{Q}\left[\cos \left(\bar{\nu}_{r} q\right)+\sin \left(\bar{\nu}_{r} q\right) / \sqrt{4 Q^{2}-1}\right] \\
& \times \exp \left(\nu_{r} q / 2 Q\right),
\end{aligned}
$$

where $\bar{\nu}_{r}=\nu_{r} \sqrt{1-1 / 4 Q^{2}}$ and $\nu_{r}=\omega_{r} \sigma_{z} / c$. The wake vanishes when $q>0$. We can easily show that the theory depends on only three dimensionless parameters, namely $\xi=I_{n} R \omega_{r}$ as a current, $\nu_{r}$ a frequency, and $Q$. In particular, the kernel is given by

$$
G_{l, m}\left(K, K^{\prime}\right)=-l \xi \operatorname{Im}\left[2 \int_{0}^{\infty} d \nu \frac{h_{l}(\nu, K) h_{m}^{*}\left(\nu, K^{\prime}\right)}{\nu \nu_{r}+i Q\left(\nu_{r}^{2}-\nu^{2}\right)}\right],
$$

which is derived using Eq. (5.8). Similarly, we have

$$
N_{l, m}^{\alpha, \beta}=\frac{-l \xi e^{-V_{\min }}}{\kappa \sqrt{2 \pi}} \operatorname{Im}\left[2 \int_{0}^{\infty} d \nu \frac{g_{l}^{\alpha}(\nu) g_{m}^{\beta^{*}}(\nu)}{\nu \nu_{r}+i Q\left(\nu_{r}^{2}-\nu^{2}\right)}\right],
$$

using Eq. (6.7).

For some values of $\nu_{r}$ in the $Q=1$ broadband resonance model, the Haissinski distribution contains two peaks as shown in Fig. 10 at a sufficiently large current. As a result, it will generate a double-well potential and thus produce two branches in the canonical transformation that is outlined in the Appendix. Mostly, the two branches are overlapping except in a region between $K_{1}$ and $K_{2}$ as illustrated in the right figure.

Empirically, we found that the region between $K_{1}$ and $K_{2}$ is small near or slightly above the threshold. This observation allows us to treat each branch as if it is a separate and independent perturbation away from the double-peak Haissinski distribution, ignoring the presence of the other branch. The threshold of the instability is therefore determined by the fastest growing mode in both branches. 

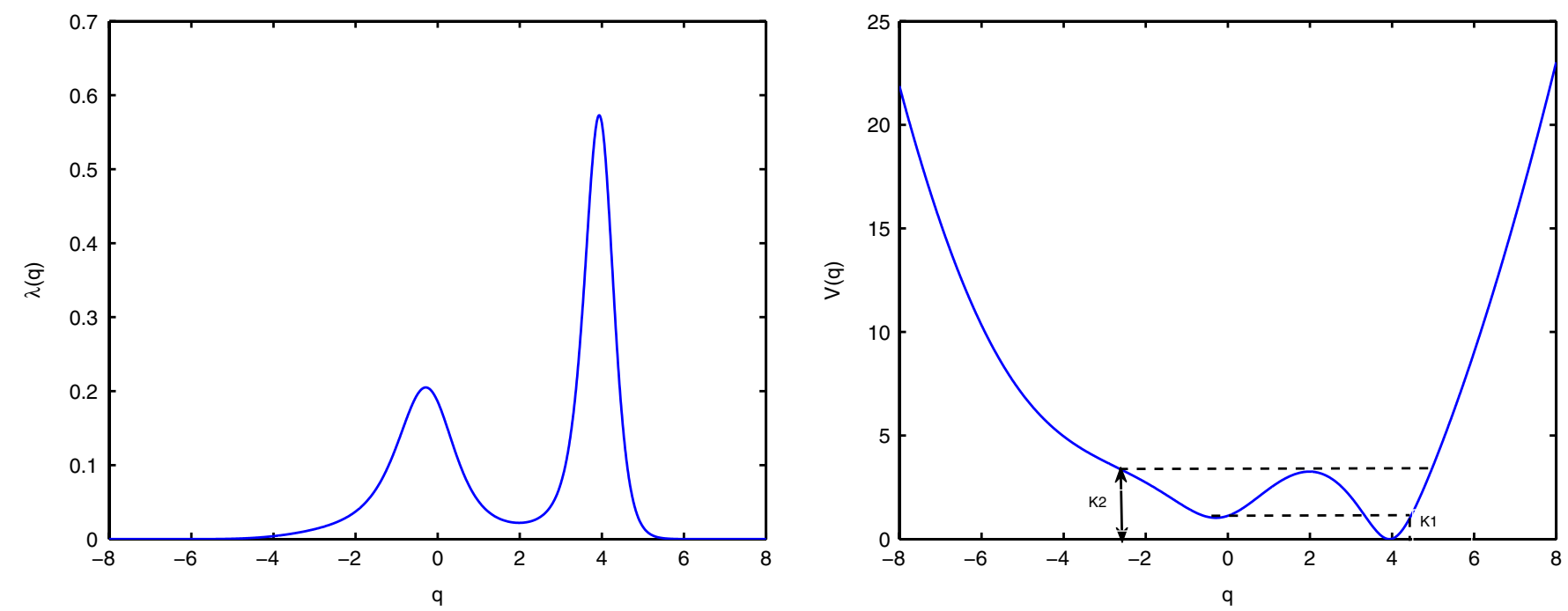

FIG. 10. Double-peak distribution on the left and its corresponding double-well potential on the right for the $Q=1$ broadband resonance model with $\nu_{r}=\omega_{r} \sigma_{z} / c=0.5$ and $\xi=I_{n} R \omega_{r}=18$.

For $Q=1$, the broadband model was first systematically studied [5] by Oide and Yokoya. Here, we repeat the calculation with the formulation developed in this paper. It is worth noting that the same difficulty of the double well is also encountered in the original method proposed by Oide and Yokoya. As we discussed in the previous section, there are some degrees of uncertainty in determining the exact threshold due to numerical noise. To minimize the effect of the noise, we determined the threshold by extrapolating from higher currents at which the noise is much less important.

The results are summarized and shown in Fig. 11. For the VFP simulations, we simply plotted the thresholds in our previously published paper [18]. As one can see from the figure, the results from the four methods (including

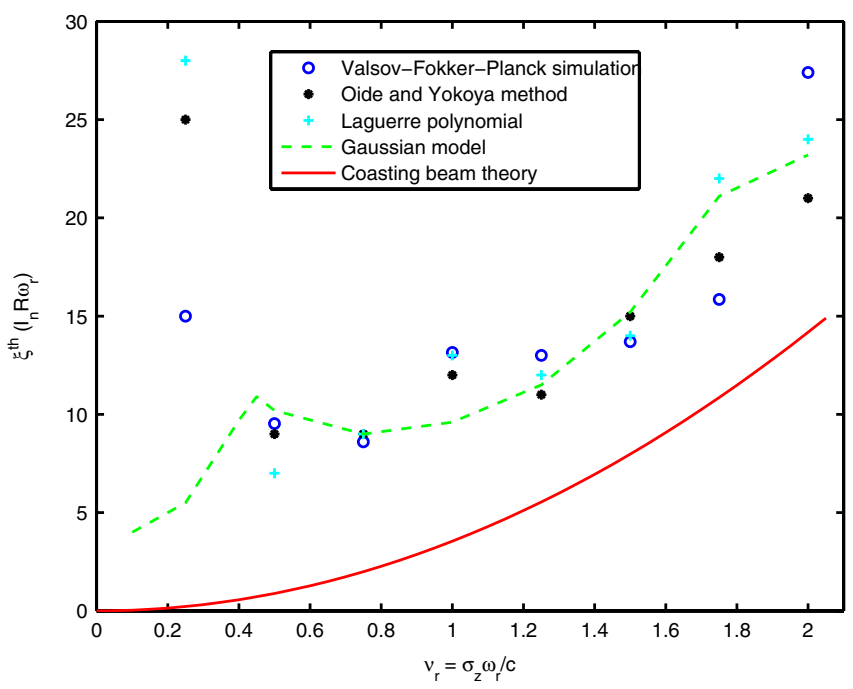

FIG. 11. Threshold as a function of $\nu_{r}=\omega_{r} \sigma_{z} / c$ in the $Q=1$ broadband impedance model with various methods.
Gaussian) are not too much different from each other. Once again, the coasting beam theory, $\xi^{\text {th }}=\sqrt{4 \pi} \nu_{r}^{2}$, provides a conservative estimate of the threshold.

\section{DISCUSSION}

We have rederived the Sacherer integral equation by linearizing the Vlasov equation and expanding it in a Fourier series in the presence of a potential-well distortion. To solve the integral equation, we employed the Laguerre polynomials and reduced it to an eigenequation of an infinite dimensional matrix. The matrix elements are written in terms of integrals that contain the incoherent spectrum and a kernel driven by impedance. This linear theory

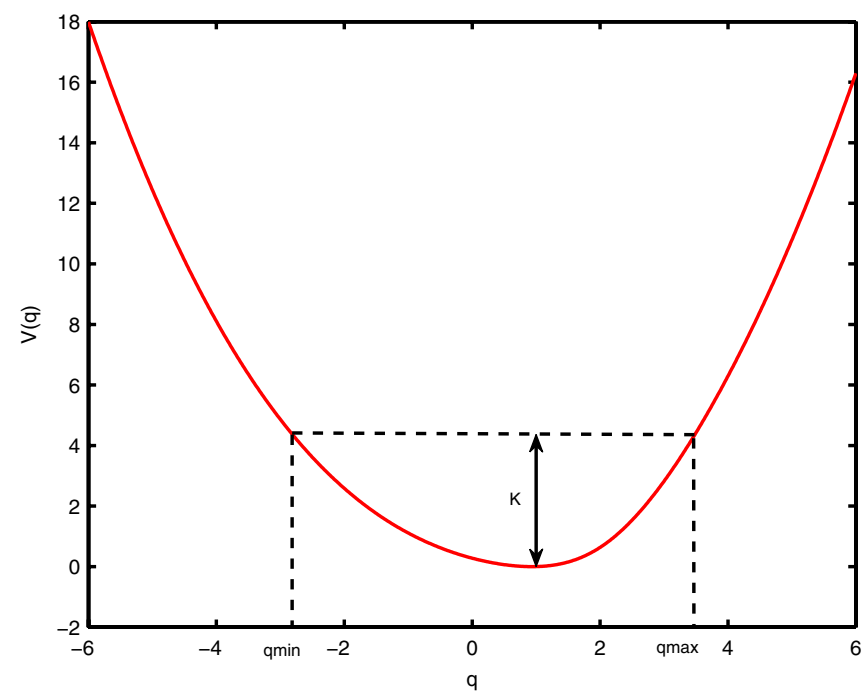

FIG. 12. The potential well at the threshold current $I_{n}^{\text {th }}=$ $0.042 \mathrm{pC} / \mathrm{V}$ in the SLC damping ring. Note that most distortion occurs near the bottom of the well. 
provides a natural and explicit extension to the modecoupling theory originated by Sacherer. For instance, with a quadratic potential $V(q)=q^{2} / 2$, it directly reduces to the well-known Gaussian model.

We applied the new method to study the longitudinal instability in the SLC damping ring; we found excellent agreements with three different methods: Laguerre polynomial, discretization, and VFP simulation. Our results also agree with the known results of the threshold. In addition, about 2 times above the threshold, the linear theory produced a dominating sextupole mode, which was observed in the measurements but not in the VFP simulations. Clearly, the linear theory provided more insights into the longitudinal dynamics in the SLC damping ring.

The method was then applied to calculate the threshold of longitudinal instability induced by CSR in free space. We found that the Vlasov theory can be characterized by a dimensional parameter $\xi=I_{n} \rho / \sigma_{z}^{4 / 3}$. The instability is caused by the coupling of two radial modes, not azimuthal ones, predicted by the Gaussian model. The coupling of the radial modes generates a much slower growth rate than the azimuthal mode coupling and therefore makes it much harder to be observed experimentally. The calculated threshold $\xi^{\text {th }}=0.50$ is in an excellent agreement with the VFP simulations. However, the discretization method gave a much lower threshold. This discrepancy remains to be resolved when computing power is further increased.

Our examples clearly show that the microwave instability is a complicated phenomenon. For a simple case such as the SLC damping, both the linear theory and simulation can provide us a reliable calculation of the threshold; sometime the linear theory may even give us more insights beyond the threshold. Mostly, the linear theory and simulation are complementary to each other. In many situations, there is an uncertainty in the calculation of the threshold due to the numerical noise or the incoherent spectrum. In spite of our improvements in the linear theory, a comprehensive theory of the microwave instability remains elusive. The key is to develop an efficient and robust method to solve the Sacherer integral equation.

\section{ACKNOWLEDGMENTS}

I greatly appreciate Bob Warnock for providing his numerical solvers for the Haissinski and the VFP equations. I would like also to thank Karl Bane, Alexander Chao, Gennady Stupakov, and Bob Warnock for many helpful and stimulating discussions. This work was supported by the Department of Energy under Contract No. DE-AC02-76SF00515.

\section{APPENDIX: CANONICAL TRANSFORMATION}

Let us assume that we have solved the Haissinski equation and found a solution of $\lambda_{0}(q)$. The unperturbed Hamiltonian $H_{0}$ can be computed according to Eq. (2.1) simply with the substitution of $\lambda(q)=\lambda_{0}(q)$. If we introduce the free energy $K=H_{0}-V_{\min }$ of a particle confined in a potential well $V(q)$, we have $K=\frac{1}{2} p^{2}+V(q)$, where $V(q)$ is given by

$$
V(q)=\frac{q^{2}}{2}-I_{n} \int_{-\infty}^{q} d q^{\prime \prime} \int_{-\infty}^{\infty} d q^{\prime} W\left(q^{\prime \prime}-q^{\prime}\right) \lambda_{0}\left(q^{\prime}\right)-V_{\min }
$$

where $V_{\min }$ is the minimum value of the static potential generated by the wakefield. For this kind of closed system, it is well known [20] that the canonical transformation to the action-angle variables can be generated by

$$
F_{2}(q, J)= \pm \int^{q} \sqrt{2\left[K(J)-V\left(q^{\prime}\right)\right]} d q^{\prime},
$$

then the angular variable $\phi$ is given by

$$
\phi=\frac{\partial F_{2}}{\partial J}= \pm \int^{q} \frac{d K / d J}{\sqrt{2\left[K(J)-V\left(q^{\prime}\right)\right]}} d q^{\prime} .
$$

To make our convention consistent with that of CourantSnyder in the case of simple harmonics, we choose $\phi$ in the range of $0 \leq \phi \leq \pi$,

$$
\frac{\omega_{s}}{\omega(K)} \phi=-\int_{q_{\max }}^{q} \frac{1}{\sqrt{2\left[K-V\left(q^{\prime}\right)\right]}} d q^{\prime},
$$

where $q_{\max }$ is one of the turning points for a particle with a given free energy $K$ inside the potential well as shown in Fig. 12. Here we have used $\omega(K) / \omega_{s}=d H_{0} / d J$ as we defined previously. Since it is a periodic system, when $\phi=$ $\pi$, it should reach the other end of the potential well and therefore we have

$$
\frac{\omega(K)}{\omega_{s}}=-\pi / \int_{q_{\max }}^{q_{\min }} \frac{1}{\sqrt{2\left[K-V\left(q^{\prime}\right)\right]}} d q^{\prime} .
$$

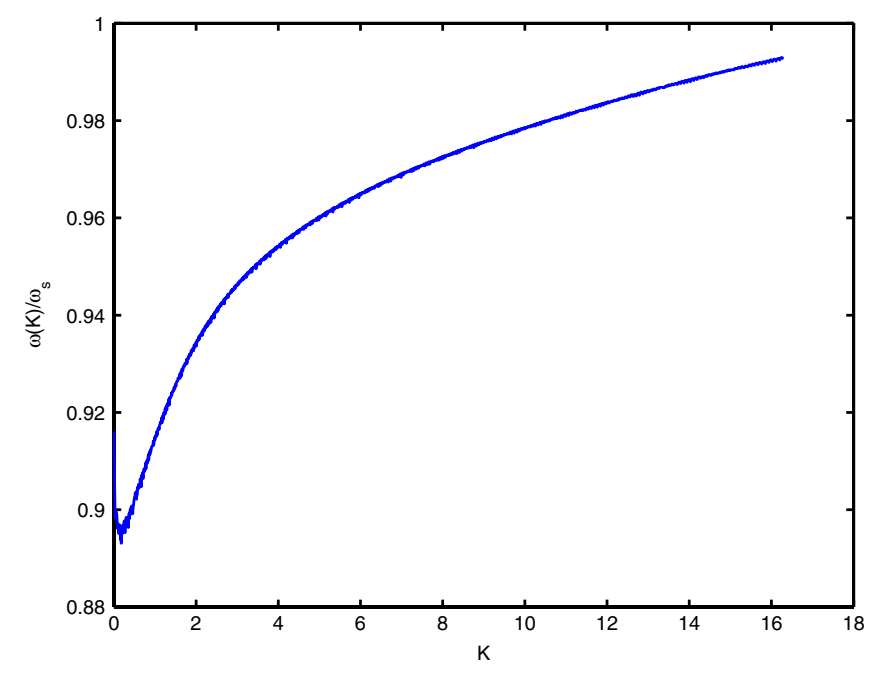

FIG. 13. Incoherent tune shift as a function of $K$ at the threshold current $I_{n}^{\text {th }}=0.0420 \mathrm{pC} / \mathrm{V}$ in the SLC damping ring. 
In Fig. 13, we plot the incoherent tune shifts due to the distorted potential at its threshold current in the SLC damping ring. It is worth noting that the tune shift mostly occurs at small amplitudes and it asymptotically vanishes at very large amplitude. As a result, an expansion of polynomials is not a good approximation of this tune shift.

Sometime we need to know $q$ as a function of $\phi$ and $K$. That can be done simply to invert the function $\phi=$ $\phi(q, K)$. For the other half of the period, $\pi \leq \phi \leq 2 \pi$, the treatment is similar, except the momentum has to be reversed. Namely,

$$
\frac{\omega_{s}}{\omega(K)}(\phi-\pi)=\int_{q_{\min }}^{q} \frac{1}{\sqrt{2\left[K-V\left(q^{\prime}\right)\right]}} d q^{\prime} .
$$

With this definition of $\phi$, it is easily shown that $q(-\phi, K)=q(\phi, K)$. This reflection symmetry allows us to simplify many calculations in the later sections.

Since there is one-to-one correspondence between $J$ and $K$, it is easy to convert the dependence from $K$ to $J$. Actually, we found that it is more convenient to use $K$ as the radial variable.

[1] F. Sacherer, IEEE Trans. Nucl. Sci. 20, 825 (1973); IEEE Trans. Nucl. Sci. 24, 1393 (1977).

[2] G. Besnier, Nucl. Instrum. Methods 164, 235 (1979).

[3] B. Zotter, CERN Reports No. SPS/81-18, No. SPS/81-19, and No. SPS/81-20 (DI), 1981.

[4] T. Suzuki, Y. Chin, and K. Satoh, Part. Accel. 13, 179 (1983).

[5] K. Oide and K. Yokoya, KEK Report No. 90-10, 1990.

[6] J. Haissinski, Nuovo Cimenta B 18, 72 (1973).
[7] K. Bane and K. Oide, in Proceedings of the Particle Accelerator Conference, Dallas, TX, 1995 (IEEE, New York, 1995), p. 3105.

[8] B. V. Podobedov, Ph.D. thesis, Stanford University [Report No. SLAC-Report-543, 1999].

[9] M. D'yachkov, Ph.D. thesis, University of British Columbia, 1995.

[10] R. Warnock, M. Venturini, and J.A. Ellison, in Proceedings of the 8th European Particle Accelerator Conference, Paris, 2002 (EPS-IGA and CERN, Geneva, 2002), p. 1589; R. Warnock, G. Stupakov, M. Venturini, and J.A. Ellison, in Proceedings of the 9th European Particle Accelerator Conference, Lucerne, 2004 (EPSAG, Lucerne, 2004), p. 2215.

[11] M. Sands, SLAC Report No. 121, 1979.

[12] Alexander W. Chao, Physics of Collective Beam Instabilities in High Energy Accelerators (John Wiley \& Sons, Inc., New York, 1993).

[13] R. Warnock and J. Ellison, Report No. SLAC-PUB-8404, 2000.

[14] K. Bane and C. Ng, in Proceedings of the Particle Accelerator Conference, Washington, DC, 1993 (IEEE, New York, 1993), p. 3432.

[15] T. Suzuki, KEK Report No. 82-34, 1983.

[16] J. B. Murphy, S. Krinsky, and R. L. Gluckstern, Part. Accel. 57, 9 (1997).

[17] A. Faltens and L.J. Laslett, Part. Accel. 4, 151 (1973).

[18] K. Bane, Y. Cai, and G. Stupakov, Phys. Rev. ST Accel. Beams 13, 104402 (2010).

[19] G. Stupakov and S. Heifets, Phys. Rev. ST Accel. Beams 5, 054402 (2002).

[20] L. D. Landau and E. M. Lifshitz, Mechanics, Course of Theoretical Physics (Pergamon Press, Oxford, 1976), Vol. 1, 3rd ed. 